\title{
Case Report: Follow Up of a Patient with De Novo Mutation at Kcna2 Gene Leading to Ataxia and Epileptic Encephalopathy
}

ISSN: 2639-0531

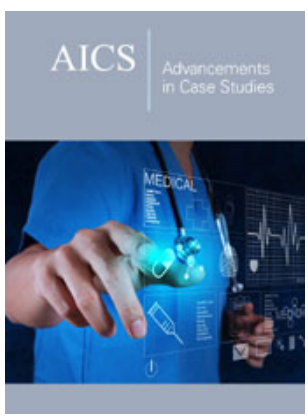

*Corresponding author: Zanluchi VBS, 1Medical Student in Pontifical Catholic University of Parana (PUCPR), Brazil

Submission: 韭 May 01, 2020

Published: 眥 June 04, 2020

Volume 2 - Issue 4

How to cite this article: Zanluchi VBS, Zanluchi LBS,Poli- Frederico RC. Case Report: Follow Up of a Patient with De Novo Mutation at Kcna2 Gene Leading to Ataxia and Epileptic Encephalopathy. Adv Case Stud. 2(4). AICS.000545. 2020. DOI: 10.31031/AICS.2020.02.000545

Copyright@ Zanluchi VBS, This article is distributed under the terms of the Creative Commons Attribution 4.0 International License, which permits unrestricted use and redistribution provided that the original author and source are credited.

\author{
Zanluchi VBS ${ }^{1 *}$, Zanluchi LBS ${ }^{2}$ and Poli- Frederico RC ${ }^{3}$ \\ ${ }^{1}$ Medical Student in Pontifical Catholic University of Parana (PUCPR), Brazil \\ ${ }^{2}$ Resident in Surgical Oncology at the Cancer Institute of Londrina (ICL), Brazil \\ ${ }^{3}$ Professor in Pontifical Catholic University of Parana (PUCPR), Brazil
}

\begin{abstract}
Background: The KCNA2 gene codifies a protein of the Kv1.2 potassium channel. Most of the last are express in the central nervous system, where they have an important role in the release of neurotransmitters and neural excitability. A mutation on this gene leads to a drastic over function with the permanent opening of the referred channel.

Case presentation: A 13- year-old male child, was diagnosed in 2014 with a mutation on the KCNA2 gene during the investigation of ataxia and epileptic encephalopathy, discovering a new cause of neurodevelopmental disorder. A detailed description of the symptoms of the patient was obtained during his lifetime until the publication of this article.
\end{abstract}

Conclusion: A new cause of neurodevelopmental disorder was found during an investigation of the epileptic encephalopathy and ataxia-the mutation in the KCNA2 gene-, as a detailed evolution of this syndrome.

Keywords: KCNA2 gene; Neurodevelopmental disorder; Epileptic Encephalopathy; Ataxia; Case report

\section{Introduction}

Most of the voltage-dependent potassium channels are expressed in the central nervous system, with an important role in the neuronal excitability and release of neurotransmitters. Mutations on the KCNA2 gene leads to hyperexcitability or electrical silencing of the neurons expressing the Kv1.2 protein [1]. The first report in the literature showing the KCNA2 gene as a cause of disease in humans was made by Pena [2]. The discovery was made after exome sequencing of a 7-year-old male child. Before that, it was known that the mutation of the same gene in murines leads to ataxia and epilepsy. It is extremely important to say that the same patient of the study published by Pena [2] is the patient of this case report.

After the prementioned publication, a series of other case reports related the mutation on the KCNA2 gene with epileptic encephalopathy and ataxia in other patients. A single study in Europe encountered this mutation in seven patients [1]. About sixteen [1-7] patients have been diagnosed with a de novo mutation in the KCNA2 gene around the world and seven patients (all members of the same family) [7] were found to have a deletion in this gene leading to a dominant autosomal pattern of inheritance causing a similar neurological syndrome but with normal intellect. Although the mutation in the KCNA2 gene been recently discovered, its functional consequences in the organism are not fully understood. Therefore, it is relevant long term follows up of the patients with this disease to better understand the manifestations of this syndrome. Thus, this case report provides a better understanding of its pathophysiology, chronological evolution, and drug treatment of the patient.

\section{Case Presentation}

Patient L.N.Z born on $08 / 07 / 2006$, prenatal was made correctly and the pregnancy had no complications. He was born after 38 weeks of cesarean section, as there was no spontaneous rupture of the amniotic pouch. The APGAR was $9-10,47 \mathrm{~cm}$ of length and $3,220 \mathrm{Kg}$, any abnormality was noted in the first neonatal exams. In the first year, he presented difficulties to 
suck, started the feed and early sleep, and this made him lost $800 \mathrm{~g}$ in the first 10 days. The pediatric recommended start complementary feeding with milk formula and was effective. The development was within the expected standard sat without support at 5 months, crawled at 7 months, at 8 months babbled, walked with support from 11 months. However, his independent march took place at 1 year and 5 months but was an ataxic gait and spoke only with 2 years and 5 months, but the mother needs to translate although he can form small phrases and this persists until now.

During the first year of life, the patient was diagnosed with asthma and have several upper air infections. At 15 months, after another episode of infection refractory to treatment, he had his first febrile seizure. After two weeks he had another seizure, but this time without fever, the mother carried to the emergency and an electroencephalogram (EEG) was made and the result was normal, but the doctor, decided to start medication for epilepsy: valproic acid $0.7 \mathrm{~mL}$ twice a day (Table 1 ). After one month, in the consult with his pediatric, another EEG was made and the result was: diffuse slowing of the baseline without foci or paroxysms. And because he was presenting fever for six days, lab exams including serologies were made and IGG was positive for Rubeola, the others were normal.

Table 1: History of therapeutic strategies already used.

\begin{tabular}{|c|c|}
\hline Age & Medications \\
\hline 15 months & Valproic acid $0.7 \mathrm{~mL}$ twice a day \\
\hline 1 year and 8 months & Valproic acid $1 \mathrm{~mL}$ twice a day \\
\hline 1 year and 10 months & Clonazepam 4 drops when crisis + Topiramate $100 \mathrm{mg} /$ day \\
\hline 2 years and 6 months & Clonazepam to 3 drops in morning and 3 in the night + valproic acid $375 \mathrm{mg} /$ day. \\
\hline 2 years and 7 months & Clobazam 30mg/day + Topiramate $9 \mathrm{mg} / \mathrm{kg} /$ day + valproic acid 375mg/day \\
\hline 4 years & Clobazam 30mg/day + Topiramate $9 \mathrm{mg} / \mathrm{kg} /$ day + valproic acid $375 \mathrm{mg} /$ day + Imipramine $10 \mathrm{mg}$ in the morning \\
\hline 6 years & Topiramate $150 \mathrm{mg} /$ day + Ethosuximide 500mg/day + Clobazam 30mg/day \\
\hline 7 years and 1 month & Clobazam $10 \mathrm{mg} /$ day (3x/day) + topiramate $100 \mathrm{mg}+50 \mathrm{mg}+50 \mathrm{mg}+$ Imipramine $10 \mathrm{mg}$ in the morning \\
\hline$?$ & Topiramate + Ethosuximide + Clobazam \\
\hline 7 years and 10 month (DIAGNOSTIC) & Acetazolamide $375 \mathrm{mg} /$ day \\
\hline 9 years and 1 month & Levetiracetam 750mg + Topiramate 50mg + Clobazam 10mg 12h/12h \\
\hline 11 years and 11 months & Levetiracetam $750+$ Topiramate $100 \mathrm{mg}+$ Clobazam $10 \mathrm{mg} 12 \mathrm{~h} / 12 \mathrm{~h}$ \\
\hline 13 years and 2 months & Levetiracetam $750 \mathrm{mg}+$ Topiramate $100 \mathrm{mg}+$ Clobazam $10 \mathrm{mg} 12 \mathrm{~h} / 12 \mathrm{~h}$ acetazolamide $250 \mathrm{mg}$ \\
\hline 13 years and 3 months until now & Levetiracetam $750 \mathrm{mg}+$ Topiramate $100 \mathrm{mg}+$ Clobazam $10 \mathrm{mg} 12 \mathrm{~h} / 12 \mathrm{~h}+$ valproic acid $250 \mathrm{mg} /$ day \\
\hline
\end{tabular}

On December 27 of 2007 (one year and five months old) the patient had another upper airway infection, bronchospasm followed by an absence crisis for a few seconds. Parents sought medical assistance, antipyretic, salbutamol, and inhalation were prescribed stabilizing the condition. On March 15 of 2008, he was diagnosed with pneumonia, and even in treatment had some seizures, the next day he had 10 crises. After the third, the patient was taken to the hospital, and the crises just stopped when Phenobarbital was administrated. The neurology that was following the case, decided to raise the dose of valproic gradually by serum dosages of the drug, arriving at the end of the month in the dose $1 \mathrm{~mL}$ twice a day. And the patient started to do respiratory physiotherapy.

After one week of physiotherapy and the clinical picture stabilizing, he had a severe asthmatic crisis arriving in the ICU with a saturation of $5 \%$ staying interned for 6 days, and in the D 4 he had 3 seizures. Five days after the discharge he made an EEG that showed diffuse projection brain irritating activity. At the end of this month, he fell off the bad and have a seizure without breath for around one minute. In May (1 year and 10 months old) he had a consult with the neuropediatric in the neurological examination was constated: general hypotonia, ataxic gait, hyporeflexia of members, Romberg positive, generalized fine extremity tremor. The family received counseling on how to deal with the seizures and started to use
Clonazepam 4 drops when crisis and substitute the valproic acid by Topiramate $100 \mathrm{mg} /$ day and physiotherapy for ataxia. He made an electroneuromyography that the result was normal.

January of 2009 ( 2 years and 6 months old) the neuropediatric adjusted the dose of Clonazepam to 3 drops in the morning and 3 in the night. During 2009, the patient didn't present serious health complications, only the crises that were until now mostly myoclonic, the absence ones became more frequent. The crises came when changed the doses of medication, or when use antibiotics, due falls or hit the head, and without an apparent reason. Was reintroduced the valproic acid 375mg/day. In 2010 (4 years old), the clinical picture stabilized. L.N.Z did an RMI that the result was normal and now the family and the neuropediatric were sure that is a functional encephalopathy and not structural. Imipramine was introduced due to a marked complaint of hyperkinetic behavior and inattention, observing improvement in attention, behavior, and sleep pattern with medication.

In 2011 the mother noted that the ataxia and epilepsy may have a connection because when the therapeutic scheme controls the seizures, his motor gets worse. And when his motor got better with physiotherapy the patient present seizures. Throughout the year, there were several myoclonic and absence crises, in addition 
to a considerable worsening of equilibrium. About the scholar development of the patient, since the 6 months in the morning he goes to a regular school and did not receive any special treatment. When he was 5 years and 9 months old the school requested a psychologic evaluation. The psychologist thought characteristics transition from sensory motor to preoperative stage. L.N.Z identifies everyday objects as equal objects, has shown difficulty in doing so with figures. Formally it's not possible to classify the level of intellectual ability, however, cognitive performance is perceived to be below the expected average. Perceptual motor development with significant delay.

In this year (2012) the neuropediatric replaces valproic acid with ethosuximide and removed imipramine, as it had decompensated and returned to myoclonic seizures, atypical absences, irritability, loss of sphincter control in addition to worsening balance and tremor, even in the presence of topiramate $(150 \mathrm{mg} /$ day), clobazam (30 mg/day) and valproic acid (375mg/day). There was a marked improvement in the level of alertness, better control of crises, but initially, it was more impulsive, explosive, and challenging.

The patient was stable for 9 months but returned to present absences, worsening of balance, frequent falls, getting hurt; topiramate $200 \mathrm{mg}$ /day, ethosuximide $500 \mathrm{mg}$ /day, and clobazam $40 \mathrm{mg}$ /day. He was emaciated, sleepy, flickering, hypotonic, without motor deficits or pyramidalisms; with clear evidence of signs of fine motor incoordination (dysmetria and dysdiadokokinesia), in addition to the referred loss of sphincter control and worsening in behavior (hyperkinetic). The neuropediatric opted for reducing the dose of clobazam $30 \mathrm{mg} /$ day, and fractioning the drugs, using them 3 times/day, in addition to returning to the use of imipramine, at a dose of $10 \mathrm{mg}$ in the morning. There was considerable improvement in behavior, attention, urinary incontinence, and remission of crises.

At 7 years and 10-month-old L.N.Z was finally diagnosed by an exome with a de novo mutation in the KCNA2 gene causing ataxia and epileptic encephalopathy [2]. At this time, he was medicated with topiramate, ethosuximide, and clobazam, but poor control of the seizures was observed and, then, those medications were stopped and it was prescribed acetazolamide based on the study of Xie et al. [8], for better control of the ataxia and epileptic seizures in murines. However, after eight months using the acetazolamide, the seizures started again and with more intensity. The treatment was changed for combination levetiracetam, topiramate, and clobazam. Good control of the seizures and ataxia was achieved, but after almost 20 months of this treatment, the patient showed a quickly worsening of the seizures (ten tonical-clonic seizures in 30 days). The medical team considered this worsening relationship with the emotional state of the patient that became very anxious in this period (close to his tenth birthday) and did not modify the treatment. Indeed, after the stress period, the patient became better.

During this period, a straight relation between the seizures and the emotional state of the patient was established. When the patient was exposed to intense emotions (anxiety, pain, sadness, anger) he showed a higher incidence of seizures. Besides that, while the patient was getting closer to puberty, he presented a higher frequency of myoclonic/absence seizures and a worsening in the muscular tonus and balance. Beyond the puberty and the emotional stress, the patient became more susceptible to worsening of the seizures when he presented muscular and mental fatigue. This has been proven in part when a 90-120 minutes sleep routine after lunch improved the global state of the patient. His family report that after a myoclonic or absence seizure, the patient became worse of the ataxia but became more cooperative and interactive - tries to paint, gets more talkative, and asks about variable things. These changes remain for 2-3 days.

L.N.Z stayed stable 2 years, and just in the June of 2018 he starts to have crisis associated with an anxious state again that improve alone, and in September the myoclonic seizures came back, progressing to absence on October this was associated with sinusitis, the mother doubled the dose of topiramate and with sinusitis resolution, the crisis stopped. Once more, the patient had an uneventful year until in July 2019 he returned to present daily absence crises, associated with myoclonic crises every 15 days, then every 3 days and finally daily being bedridden for 40 days. In September and October, the use of acetazolamide $250 \mathrm{mg}$ /day was resumed without response, being replaced by valproic acid $250 \mathrm{mg} /$ day, but the seizures just ceased in November. Finally, it is a case of ataxia and epileptic encephalopathy with myoclonic and absence crises of difficult control, with psycho-emotional factors involved. Puberty and fatigue are probably worsening conditions.

\section{Discussion}

After being diagnosed with epilepsy, the patient was medicated with acetazolamide for eight months. This medication is a diuretic which mechanism of action is to inhibit the carbonic anhydrase enzyme used to reduce the intraocular pressure, edema, and as an anticonvulsant [9]. It is indicated by the Food and Drug Administration (FDA) for centrencephalic epilepsy, such as absence seizures, myoclonic, atonic; but, this drug had shown good results for treatment for other types of epilepsy, such as generalized tonicclonic and mixed seizures [10]. The first report of the use of this drug for this goal was in 1956 by Anssel et al. [11] when 26 patients with different idiopathic epilepsy were treated with $1-14 \mathrm{mg} / \mathrm{kg}$ of acetazolamide taken in two doses a day. Close to $53 \%$ of the patients showed excellent/good response, 31\% showed some therapeutic improvement. However, there was a need for increases in dosage throughout therapy.

Forsythe et al. [12] accompanied 54 pediatric patients with grand mal seizure refractory to carbamazepine treated with $10 \mathrm{mg} /$ $\mathrm{kg} /$ day acetazolamide. In 2 years follow up, 16 (40\%) of the patients reached complete remission of the seizures, $7(18 \%)$ reached $3 / 4$ of reduction, and 1 (3\%) reduced by half. Another 48 patients [13] with refractory partial seizures were reviewed retrospectively. The adjunct acetazolamide started at a dose of $3-6 \mathrm{mg} / \mathrm{kg} /$ day e where titled until a maintenance dose based on the clinical response and side effects $(3,8-22 \mathrm{mg} / \mathrm{kg} /$ day $)$. The treatment time lasted from 15 to 30 months. 21 of 48 patients (43\%) had at least a reduction of $50 \%$ in the number of seizures for the first 3 months. With the 
increasing dose, almost $80 \%$ of the patients achieved this response, with an average duration of response of 12.9 months (range: 3 to 30 months). Thus, the fact of the patient had good control of the seizures for only eight months with the Acetazolamide was expected.

According to a study by the Department of Neurology at the State University of Campinas (Unicamp), there is no higher incidence of depression or anxiety in the group of teenagers with epilepsy compared to the control group by seeing the diagnostic scores of both [14]. But it was observed that there is a lower frequency of seizures when there are low scores of trait or states of anxiety. The perception of crisis control (psychological variable) was positively correlated with low trait scores or a state of anxiety. Regarding the occurrence of crises in public places (social aspect), the correlations showed positive results between high scores of depression and traces of anxiety and the occurrence of crises in public places. Also, a study by the Texas A\&M University Health Sciences Center which investigated the neuroendocrine basis of gender differences in epilepsy claims that it has already been clarified that changes in seizure sensitivity are also evident at puberty, which is associated with too rigorous changes in reproductive hormones and behavioral patterns. Testosterone is known to produce both pro-convulsive and anticonvulsant behavior depending on the animal and the type of seizure. Both animal and clinical studies show that testosterone enhances seizure activity by metabolism to estrogens [15].

About the genetic aspects of this case report, the patient has a single nucleotide polymorphism (SNP) involving the change c. $890 \mathrm{G}>\mathrm{A}$, leading to a replacement of the amino acid p.Arg $297 \mathrm{Gln}$, which comprises the second of the critical arginine in the $S 4$ voltage sensor of the KV1.2 channel. A study carried out in Europe after the first publication of this case diagnosed six more patients with this mutation. One of the patients from that study has the same mutation as the patient of the present article, and it was investigated how this substitution changes the channel. It was discovered that this mutation increased the current amplitudes by nine times and changed the voltage dependence of the steady-state activation by $-40 \mathrm{mV}$ compared to the wild-type channel. It was also found that carriers of mutations with dominant effects of function gain were more severe in terms of epilepsy, ataxia, and intellectual disability and also differed electrographically, with generalized epileptic discharges [1].

Furthermore, in the Kv1.1 subfamily (the same channel family as this patient), mutations are already known to cause ataxia and epileptic encephalopathy and have been more explored. A review made by D'Adamo et al. [16] summarize all the mutations and therapeutic approaches already studied in the KCNA1 channel. In this article two potential therapeutic targets for remittent seizures caused by loss-of-function changes in Kv1 channels were identified: non-inactivating or persistent sodium channels and N-methyl-Daspartate (NMDA) receptor. This hypothesis is based on the finding that a small inhibition of persistent sodium channels and NMDA block was sufficient to reduce firing rate, decrease action potential duration, and glutamate release, all altered after simulated Kv1.1 and Kv1.2 loss-of-function. Therefore, the repurposing of riluzole (a sodium channel and NMDA receptor blocker and BK channel activator), is suggested as a treatment for epilepsy caused by LGI1, KCNA1, or KCNA2 mutations [16].

\section{Conclusion}

Finally, the case reported, and the articles bring to light the comprehension of the evolution of a presentment of a new genetic mutation and provide a better understanding of its pathophysiology, chronological evolution, and drug treatment of the patient. Our goal is to assist the development of treatments and serve as a reference for other medical assistants and families with individuals with this condition.

\section{Acknowledgment}

Zanluchi, VBS was responsible for data collection and writing. Zanluchi, LBS was responsible for writing assistance and translation. Poli-Frederico, RC is our professor, was our tutor, guide, and reviewer.

\section{References}

1. Syrbe S, Hedrich UBS, Djémié T, Riesch E, Muller S, et al. (2015) De novo loss- or gain-of-function mutations in KCNA2 cause epileptic encephalopathy. Nat Genet 47(4): 393-399.

2. Pena SDJ, Coimbra RLM (2014) Ataxia and myoclonic epilepsy due to a heterozygous new mutation in KCNA2: proposal for a new channelopathy. Clinical Genetics 87(2): e1-e3.

3. Pu Miao, Feng J, Guo YF, Wang J, Xiao Xu, et al. (2018) Genotype and phenotype analysis using an epilepsy-associated gene panel in Chinese pediatric epilepsy patients. Clin Genet 94(6): 512-520.

4. Hundallah K, Alenizi A, Alhashem A, Tabarki B (2016) Severe earlyonset epileptic encephalopathy due to mutations in the KCNA2 gene: expansion of the genotypic and phenotypic spectrum. Eur J Paediatr Neurol 20(4): 657-660.

5. Allen NM, Conroy J, Shahwan A, Lynch B, Correa RG, et al. (2015) Unexplained early onset epileptic encephalopathy: exome screening and phenotype expansion. Epilepsia 57(1): 12-17.

6. Allou L, Julia S, Amsallem D, El Chehadeh S, Lambert L, et al. (2017) Rettlike phenotypes: expanding the genetic heterogeneity to the KCNA2 gene and first familial case of CDKL5-related disease. Clin Genet 91(3): 431-440.

7. Corbett MA, Bellows ST, Li M, Carroll R, Micallef S, et al. (2016) Dominant KCNA2 mutations causes episodic ataxia and pharmaco-responsive epilepsy. Neurology 87(19): 1975-1984.

8. Xie G, Harrison J, Clapcote SJ, HuangY, Zhang JY, et al. (2010) A new Kv1.2 channelopathy underlying cerebellar ataxia. J Biol Chem 285(42): 32160-32173.

9. Hamada K, Song H, Ishida S, Yagi K, Seino M (2001) Contrasting effects of zonisamide and acetazolamide on amygdaloid kindling in rats. Epilepsia 42(11): 1379-1386.

10. Turner AL, Perry MS (2017) Outside the box: Medications worth considering when traditional antiepileptic drugs have failed. Seizure 50: 173-185.

11. Ansell B, Clarke E (1956) Acetazolamide in the treatment of epilepsy. Br Med J 1(4968): 650-654.

12. Forsythe WI, Owens JR, Toothill C (1981) Effectiveness of acetazolamide in the treatment of carbamazepine-resistant epilepsy in children. Dev Med Child Neurol 23(6): 761-769. 
13. Oles KS, Penry JK, Cole DL, Howard G (1989) Use of acetazolamide as an adjunct to carbamazepine in refractory partial seizures. Epilepsia $30(1)$ : 74-78.

14. Siqueira NF, Oliveira Fernando LBB, Siqueira JA, Souza EA (2015) In adolescents with epilepsy, high scores of anxiety and depression are associated with occurrence of seizures in public places. Arq Neuropsiquiatr 73(3): 205-211.
15. Samba Reddy D (2017) The neuroendocrine basis of sex differences in epilepsy. Pharmacol Biochem Behav 152: 97-104.

16. D’adamo MC, Liantonio A, Rolland JF, Pessia M, Imbrici P (2020) Kv1.1 Channelopathies: pathophysiological mechanisms and therapeutic approaches. Int J Mol Sci 21(8): 2935.

For possible submissions Click below:

Submit Article 